CASE REPORT

\author{
J.G. Seol \\ V.A. Livolsi \\ B.W. O’Malley, Jr \\ J.Y. Chen \\ L.A. Loevner
}

\title{
Respiratory Epithelial Adenomatoid Hamartoma of the Bilateral Olfactory Recesses: A Neoplastic Mimic?
}

SUMmARY: REAH is a rare benign lesion of the sinonasal tract. The nasal cavity, particularly the posterior nasal septum, is the most common site of involvement. It usually occurs unilaterally and can be cured with conservative surgical resection. We present an unusual case of adenomatoid hamartoma involving bilateral olfactory recesses and discuss the importance of distinguishing this from other neoplastic processes that may lead to overly aggressive treatment.

ABBREVIATIONS: H\&E = hematoxylin-eosin; IP = inverted papilloma; REAH = respiratory epithelial adenomatoid hamartoma; SNAC = sinonasal adenocarcinoma

A hamartoma is a malformation of tissue that is indigenous to the involved anatomic site. It has no capacity for unimpeded proliferation and causes symptoms mainly related to mass effect on adjacent structures. Hamartomas can occur anywhere in the body, but involvement of the head and neck is exceedingly uncommon.

A particular subset known as REAH was first characterized in 1995 and represents a rare lesion of the sinonasal tract. ${ }^{1}$ It is important to distinguish REAH from more aggressive neoplastic processes because REAH is a benign entity that can be cured with conservative surgical resection.

\section{Case Report}

An otherwise healthy 60 -year-old woman presented with headaches of 2 weeks' duration. She also complained of nasal congestion, an altered sense of smell, and stuffiness in both ears. Her medical history was notable for environmental allergies and chronic sinusitis. Findings of physical examination and laboratory work-up were unremarkable.

MR and CT imaging were performed, which revealed an enhancing soft-tissue mass in the anterior left nasal cavity involving the olfactory recess and a similar-appearing smaller mass in the right olfactory recess (Fig $1 A-C$ ). There were no destructive bone changes or intracranial extension. The uninvolved paranasal sinuses were clear. Imaging findings were most consistent with a primary neoplasm of the sinonasal cavity.

Endoscopic biopsy revealed a REAH, which was subsequently resected endoscopically. On examination, the masses involved the anterior frontal skull base and bilateral olfactory recesses, left greater than right, and extended to the root of the middle turbinate. The masses were completely excised by using an endoscopic approach.

The masses measured 1.5 (left) and $0.8 \mathrm{~cm}$ (right) and were microscopically similar (Fig $1 D,-E$ ). They contained many enlarged

Received April 14, 2009; accepted after revision May 17

From the Departments of Radiology (J.G.S., L.A.L., J.Y.C.), Otorhinolaryngology (L.A.L., B.W.O.), and Pathology and Laboratory Medicine (V.A.L.), University of Pennsylvania, Philadelphia, Pennsylvania.

Julia G. Seol is a participant in the National Institutes of Diabetes and Digestive and Kidney Diseases Short-Term Education Program for Underrepresented Persons (5-T35-DK-60441-08).

Please address correspondence to Laurie A. Loevner, MD, Department of Radiology, Hospital of the University of Pennsylvania, 2 Dulles Building, 3400 Spruce St, Philadelphia, PA 19104; e-mail: laurie.loevner@uphs.upenn.edu

DOI 10.3174/ajnr.A1755 glands lined by ciliated respiratory epithelium. The periglandular connective tissue was attenuated and fibrotic, consistent with basement membrane thickening. Intervening stroma resembled that of inflammatory sinonasal polyps (ie, loose connective tissue with edema), vascular congestion, and moderate numbers of chronic inflammatory cells. Stains for $\beta$ catenin and smooth muscle actin showed positivity in the stromal cells, identical to those of a typical sinonasal polyp. Stains for S-100, c-kit, and CD34 were negative in the stromal cells. Notably, lobulated seromucinous glands were absent. These characteristic histologic findings established the diagnosis of REAH.

\section{Discussion}

Hamartomas are due to inborn errors of tissue development and are characterized by excessive proliferation of cellular components endogenous to the given tissue. ${ }^{2}$ They are composed of mature elements with no malignant potential and thus have self-limited growth. ${ }^{1}$ Hamartomas may arise in any site, most commonly originating from the lung, kidney, and intestines. Head and neck involvement is uncommon, and published studies are limited to case reports.

$\mathrm{REAH}$ is a distinct and rare type of hamartoma occurring in the sinonasal tract. Including the initial 31 cases described by Wenig and Heffner, ${ }^{1}$ there are currently approximately 50 REAH cases documented in the English literature. Most cases have occurred in adult men, and associations with tobacco use, asthma, and nasal polyposis have been noted. ${ }^{1,3-5}$ Most patients have isolated disease of the nasal cavity, though sinus involvement with or without nasal disease has been reported. ${ }^{1,5-7}$ Within the nasal cavity, REAH preferentially involves the posterior nasal septum and usually occurs unilaterally. ${ }^{1}$

Imaging studies frequently reveal an expansile soft-tissue mass connected to the nasal septum with opacification of adjacent sinuses. ${ }^{4-6,8}$ Calcification may be visualized within the lesion, but bone erosion and intracranial invasion are uncommon. ${ }^{6} \mathrm{MR}$ imaging findings of REAH include a mass that is hypo- to isointense relative to brain on T1-weighted images with a variable pattern of enhancement. ${ }^{7,8}$

Our case showed the typical radiologic findings of REAH, but the hamartoma atypically arose at bilateral olfactory recesses. Diagnostic considerations based on the clinical presentation and imaging findings included neoplasms, IP, and inflammatory polyposis. While bone preservation may not be 

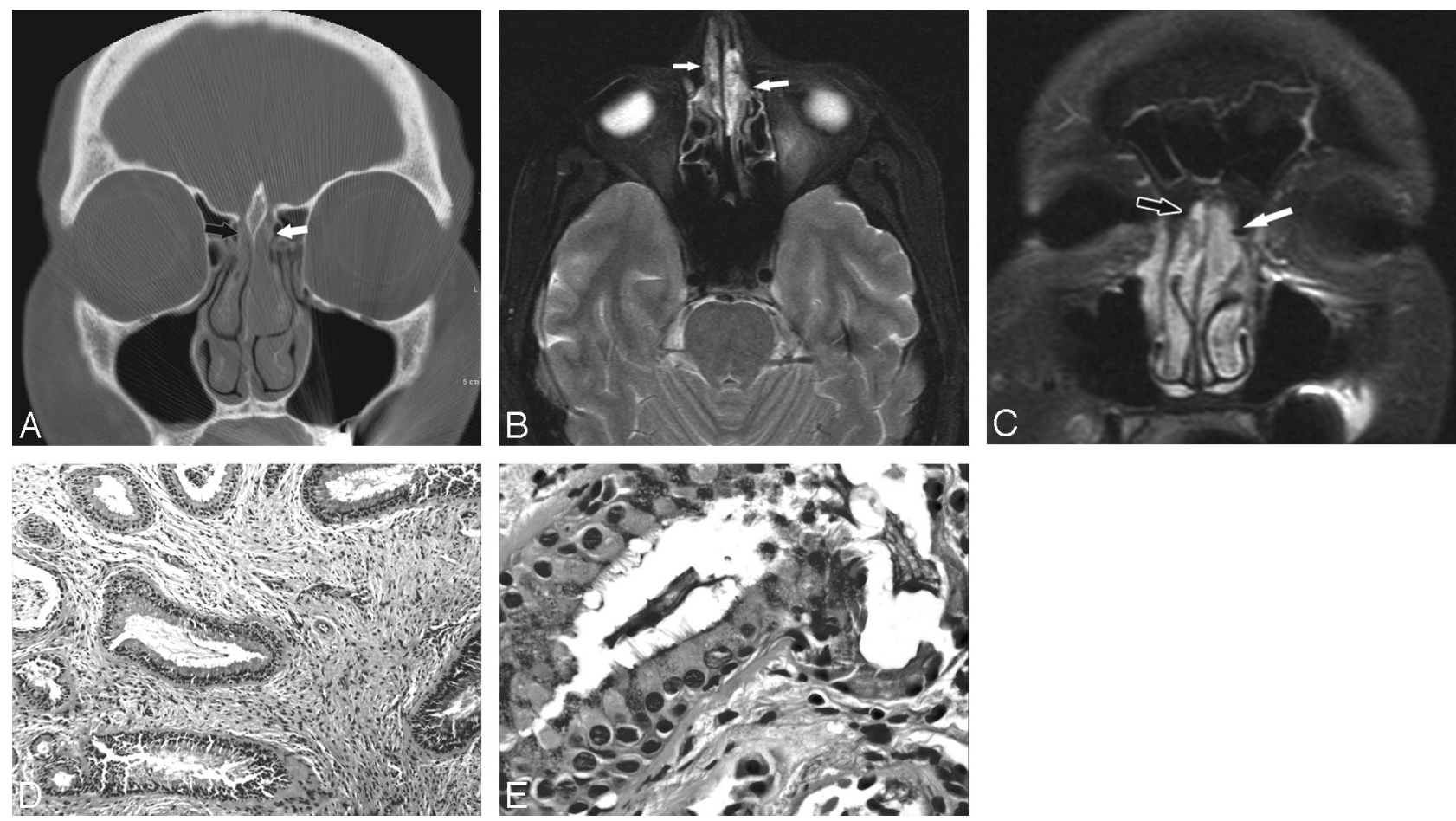

Fig 1. A 60-year-old woman with REAH of the bilateral olfactory recesses. $A$, Coronal CT image with a bone reconstruction kernel shows a mass in the upper left nasal vault filling the olfactory recess (white arrow) with mild lateral displacement of the middle turbinate. A smaller mass is also noted in the right olfactory recess (black arrow). The cribiform plate is intact. $B$, Axial T2-weighted MR image shows a heterogeneous but hyperintense mass in the left olfactory recess, which is greater than that in the right olfactory recess (arrows). $C$, Postcontrast coronal T1-weighted MR image shows that the mass mildly enhances in the left olfactory recess (white arrow) and a smaller mass enhances in the right olfactory recess (black arrow). $D$, Low-power photomicrograph of a REAH shows large slightly dilated glands within a loose connective tissue stroma with scattered inflammatory cells (H\&E, 100 $\times$ ). E, Photomicrograph of mucinous epithelial cells with cilia that project into the lumen of the gland (H\&E, 400X).

useful in differentiating REAH from polyps, the absence of destructive bone changes - as in our case-may help distinguish REAH from adenocarcinoma, esthesioneuroblastoma, and IP. Additionally, the patient's age and the location of the lesion excluded nasal glioma, and preservation of the cribriform plate excluded the diagnosis of encephalocele.

Grossly, REAH appears as an edematous polypoid mass resembling an inflammatory polyp. Characteristic histopathologic features include the proliferation of glands that originate from surface epithelium, are lined by ciliated respiratory epithelium, and are surrounded by fibrosis in an edematous stroma. ${ }^{1}$ These proliferations are directly connected to the surface epithelium and are not derived from seromucous glands, as seen in the setting of reactive hyperplasia associated with inflammatory polyps. The epithelial cells show no dysplasia or atypia, and the epithelium unrelated to the adenomatoid proliferations is normal.

In contrast to IP, REAH involves respiratory epithelium that forms glands in a single layer. Inverted papilloma arises from the invagination of surface squamous epithelium into underlying stroma and is characterized by a thickened epithelium, local invasion, and bony erosion. ${ }^{2}$

Recently, Weinreb et $\mathrm{al}^{9}$ described 7 cases of sinonasal seromucinous hamartoma with histopathology ranging from pure seromucinous to mixed seromucinous/REAH features. Seromucinous hamartoma is distinguished from REAH by its proliferation of glands in a lobular, clustered, or haphazard arrangement. However, both are covered by a ciliated respiratory-type epithelium, share similar stromal features, and commonly arise in similar locations, suggesting that seromucinous hamartoma and REAH may exist on a spectrum of sinonasal hamartomas rather than as separate entities.

The etiology of REAH is unclear but is thought to involve an inflammatory process. This hypothesis arises from the observed occurrence of REAH in the setting of rhinosinusitis, inflammatory polyposis, and sinus surgery. ${ }^{1,3,4}$ Our case was associated with allergic inflammation and chronic sinusitis, supporting the idea that inflammation may be a contributing factor in the development of REAH.

Generally, SNAC can be differentiated from REAH by its complex glandular growth pattern, cellular atypia, pleomorphism, increased mitotic figures, and lack of intervening connective tissue. ${ }^{1}$ However, recent studies suggest that REAH may be part of a hamartoma-adenoma-adenocarcinoma pathogenetic sequence or spectrum. Molecular analyses of REAH have revealed allelic loss rates higher than expected for a non-neoplastic entity but less than that of SNAC. ${ }^{10}$ Additionally, Jo et $\mathrm{al}^{11}$ described 6 cases of low-grade nonintestinal adenocarcinoma associated with REAH, in which the SNACs were admixed with glandular structures identical to those seen with REAH.

These findings raise the possibility that REAH may be classified histologically as a benign neoplasm rather than a hamartoma. However, the clinical significance of this classification is unknown, especially given the paucity of information regarding REAH, and further study of additional cases is warranted. REAH does not progress, nor does it regress spontaneously. Conservative surgical resection is the treatment of choice, and no recurrent, persistent, or metastatic disease has been reported to date. Despite its rarity, REAH should be included in 
the differential diagnosis of a symptomatic sinonasal mass because misinterpretation of this benign lesion may lead to unnecessarily aggressive surgical intervention.

\section{References}

1. Wenig BM, Heffner DK. Respiratory epithelial adenomatoid hamartomas of the sinonasal tract and nasopharynx: a clinicopathologic study of 31 cases. Ann Otol Rhinol Laryngol 1995;104:639-45

2. Melroy CT, Senior BA. Benign sinonasal neoplasms: a focus on inverting papilloma. Otolaryngol Clin North Am 2006;39:601-17

3. Liang J, O'Malley BW Jr, Feldman M, et al. A case of respiratory epithelial adenomatoid hamartoma. Am J Otolaryngol 2007;28:277-79

4. Delbrouck C, Fernandez Aguilar S, Choufani G, et al. Respiratory epithelial adenomatoid hamartoma associated with nasal polyposis. Am J Otolaryngol 2004;25:282-84
5. Ingram WF, Noone MC, Gillespie MB. Respiratory epithelial adenomatoid hamartoma: a case report. Ear Nose Throat J 2006;85:190-92

6. Athre R, Ducic Y. Frontal sinus hamartomas. Am J Otolaryngol 2005;26:419-2

7. Himi Y, Yoshizaki T, Sato K, et al. Respiratory epithelial adenomatoid hamartoma of the maxillary sinus. J Laryngol Otol 2002;116:317-18

8. Endo R, Matsuda H, Takahashi M, et al. Respiratory epithelial adenomatoid hamartoma in the nasal cavity. Acta Otolaryngol 2002;122:398-400

9. Weinreb I, Gnepp DR, Laver NM, et al. Seromucinous hamartomas: a clinicopathological study of a sinonasal glandular lesion lacking myoepithelial cells. Histopathology 2009;54:205-13

10. Ozolek JA, Hunt JL. Tumor suppressor gene alterations in respiratory epithelial adenomatoid hamartoma (REAH): comparison to sinonasal adenocarcinoma and inflamed sinonasal mucosa. Am J Surg Pathol 2006;30:1576-80

11. Jo VY, Mills SE, Cathro HP, et al. Low-grade sinonasal adenocarcinomas: the association with and distinction from respiratory epithelial adenomatoid hamartomas and other glandular lesions. Am J Surg Pathol 2009;33:401-08 